\title{
Downregulation of hepatoma-derived growth factor suppresses the malignant phenotype of U87 human glioma cells
}

\author{
AIXIA ZHANG ${ }^{1}$, WEIGUO LONG ${ }^{2}$, ZE GUO $^{3}$, ZHENYING GUO $^{3}$ and BRIAN B. CAO ${ }^{4}$ \\ ${ }^{1}$ School of Pharmacy, Nanjing Medical University, Nanjing; ${ }^{2}$ Department of Pathology, Affiliated Hospital \\ of Jiangsu University, Zhenjiang 212001; ${ }^{3}$ School of Basic Medical Sciences, Nanjing Medical University, \\ Nanjing 210029, P.R. China; ${ }^{4}$ Van Andel Research Institute, Grand Rapids, MI 49503, USA
}

Received January 13, 2012; Accepted March 12, 2012

DOI: $10.3892 /$ or.2012.1768

\begin{abstract}
Hepatoma-derived growth factor (HDGF) has been shown to correlate with increased malignancy of different types of tumors and could be an independent prognostic index for human cancers. We previously found that HDGF is overexpressed in glioma tissues and that its expression level may correlate with the clinical pathological grade. In the present study, we investigated the effects of HDGF downregulation on the biological behaviors of U87 glioma cells. Our results showed that HDGF knockdown significantly inhibited the malignant phenotype of U87 cells, including the colony formation, migration and invasion in vitro, as well as tumorigenesis in vivo. Our data also suggest that hepatocyte growth factor/scatter factor (HGF/SF) may contribute to the HDGFassociated aggressive behavior of glioma cells.
\end{abstract}

\section{Introduction}

Malignant gliomas are the most common type of primary brain tumor in adults (1). Even with advances in surgery, radiation therapy, and chemotherapy, the median survival of patients with malignant gliomas has not changed substantially in the last 20 years $(2,3)$. Understanding the detailed mechanisms behind the biological features of this disease may provide new options for molecularly targeted therapies.

Hepatoma-derived growth factor (HDGF) was originally purified from the conditioned medium of a human hepatoma cell line and showed a proliferative effect on fibroblasts (4). Although HDGF has been demonstrated to have mitogenic

Correspondence to: Dr Brian B. Cao, Van Andel Research Institute, 333 Bostwick Avenue, N.E., Grand Rapids, MI 49503, USA E-mail: brian.cao@vai.org

Dr Aixia Zhang, School of Pharmacy, Nanjing Medical University, Nanjing 210029, P.R. China

E-mail: aixiazh@gmail.com

Key words: hepatoma-derived growth factor, glioma, migration, in vitro invasion activity in endothelial cells, vascular smooth muscle cells, and cancer cells (5), the biological functions of HDGF are still largely unknown. HDGF is highly expressed in the early stages of development in the brain, kidney, liver, and cardiovascular system, but it decreases rapidly after birth, suggesting that it plays an important role in tissue growth and differentiation (6-9).

Accumulating clinical evidence demonstrates that HDGF expression is correlated with increased malignancy of different types of tumors and that it could be an independent prognostic factor in a variety of human cancers (10-13). We recently found that HDGF is overexpressed in glioma specimens, and its expression level may correlate with the clinical pathological grade (14). However, it remains unclear how HDGF affects the biological behavior of glioma cells. In the present study, we knocked down HDGF using small interfering RNA (siRNA) and investigated the effects of its downregulation on the malignant phenotype of U87 glioma cells. Our results showed that the downregulation of HDGF significantly inhibited the colony formation, migration, and invasion of U87 cells in vitro, as well as tumorigenesis in vivo. Microarray data indicated that HDGF may be involved in glioma progression via the regulation of hepatocyte growth factor/scatter factor (HGF/SF).

\section{Materials and methods}

Cell lines and animals. The human glioma cell lines U118, SW1783, DBTRG, U87 and U373; the hepatoblastoma cell line HepG2; the epithelial carcinoma cell line A431; and the mouse NIH 3T3 fibroblasts were maintained in DMEM supplemented with $10 \%$ fetal bovine serum (FBS). All cell lines were cultured in a humidified incubator at $37^{\circ} \mathrm{C}$ with $5 \% \mathrm{CO}_{2}$.

BALB/c nude mice (Shanghai Laboratory Animal Center, CAS, Shanghai, China) were bred and maintained in a standard pathogen-free mouse colony of the Center of Experimental Animals at Nanjing Medical University. Animal experiments were performed in compliance with the guidelines of the University Ethics Committee for Laboratory Animal Research.

Reagents and antibodies. Lipofectamine 2000 was purchased from Invitrogen (Carlsbad, CA). 3-(4,5-Dimethylthiazol-2-yl)2,5-diphenyltetrazolium bromide (MTT) was obtained from Promega (Madison, WI). Matrigel was obtained from BD 
Biosciences (San Jose, CA). Millicell cell culture inserts were purchased from Millipore (Billerica, MA). HDGF and HGF/ $\mathrm{SF}$ monoclonal antibodies (mAbs) were generated as previously described $(14,15)$.

Transient transfection. Two small interfering RNAs (siRNAs) specific for HDGF were synthesized (GenePharma Co., Ltd., Shanghai, China) according to published data (16). We also synthesized a scrambled RNA which mismatches to any human gene as the negative control. U87 glioma cells were transfected with siRNA using Lipofectamine following the manufacturer's instructions and were cultured for $48 \mathrm{~h}$ before use.

RT-PCR and western blot analysis. Total RNA was extracted from the experimental cells using TRIzol reagent (Invitrogen) according to the manufacturer's protocol and then was reversetranscribed into cDNA. PCR amplification was carried out for 35 cycles of $94^{\circ} \mathrm{C}$ for $1 \mathrm{~min}, 60^{\circ} \mathrm{C}$ for $1 \mathrm{~min}$, and $72^{\circ} \mathrm{C}$ for 2 min before a 5-min initial denaturation at $94^{\circ} \mathrm{C}$ and followed by incubation at $72^{\circ} \mathrm{C}$ for $10 \mathrm{~min}$. The primer pairs specific for HDGF (724 bp) were 5'-GACAGCAGCAGGAACAGT-3' (forward) and 5'-GCCATGTCGCGATCCAAC-3' (reverse). $\mathrm{HGF} / \mathrm{SF}$ (600 bp) was amplified using the following primers: 5'-AAGGACGCAGCTACAAGGGAAC-3' (forward) and 5'-GAGAATCCCAACGCTGACATGGA-3' (reverse).

For western blots, protein extracts were prepared from the cell lines using RIPA buffer (20 mM Tris-HCl, $\mathrm{pH} 7.4$, $1 \% \mathrm{NP}-40,150 \mathrm{mM} \mathrm{NaCl}$ ), were separated by SDS-PAGE, and then were transferred to a nitrocellulose membrane. The membrane was blocked with $5 \%$ milk for $2 \mathrm{~h}$, followed by incubation with the anti-HDGF mAb (1:1000) or anti-HGF/ $\mathrm{SF} \mathrm{mAb}$ (1:1000). The immune-reactive band was detected using a goat anti-mouse IgG horseradish peroxidase conjugate (1:2000, Sigma) and an ECL kit (Pierce, Rockford, IL).

Cell proliferation assay. In vitro cell proliferation was assessed in a Lipofectamine-treated group and an HDGF-siRNA-1treated group. In each group, cells were distributed into 96-well plates at a density of $5 \times 10^{3}$ per well in serum-free DMEM and incubated for $48 \mathrm{~h}$. To determine the number of cells, $20 \mu \mathrm{l}$ of MTT reagent was added to each well, and the absorbance was read at $492 \mathrm{~nm}$ after $4 \mathrm{~h}$ of incubation. Experiments were performed three times in quintuplicate.

Soft agar colony formation. Forty-eight hours after transfection, 2,000 cells/well were seeded in 6-well plates in triplicate with a bottom layer of $0.6 \%$ low-melting agar and a top layer of $0.3 \%$ agar in DMEM. The plates were covered with $1 \mathrm{ml}$ of DMEM containing $10 \% \mathrm{FBS}$ and incubated at $37^{\circ} \mathrm{C}$. After two weeks, the numbers of colonies with $>50$ cells were counted using an inverted microscope with x100 magnification.

Cell migration and in vitro invasion assay. Millicell cell culture inserts with $8-\mu \mathrm{m}$ pore filters for 24-well plates were used in the migration assay according to the manufacturer's instructions. Briefly, $2 \times 10^{4}$ cells in $200 \mu 1$ of serum-free medium were added into the upper chamber and then placed into wells containing $800 \mu \mathrm{l}$ of NIH 3T3 conditioned medium in the lower chamber (17). After incubation for $6 \mathrm{~h}$ at $37^{\circ} \mathrm{C}$, cells on the top of the filters were gently wiped off with a cotton swab. Migrated cells on the bottom side were fixed, stained, and counted under a microscope in 5 randomly selected fields at x200 magnification. Three independent experiments were performed, each of them in duplicate.

For the cell invasion assay, the upper side of the porous membrane was coated with Matrigel (1:10 dilution with DMEM) at $37^{\circ} \mathrm{C}$ overnight and then was hydrated with $100 \mu \mathrm{l}$ of pre-warmed DMEM. Cells $\left(4 \times 10^{4}\right)$ were seeded into each of the upper compartments and incubated in serum-free medium at $37^{\circ} \mathrm{C}$ for $24 \mathrm{~h}$. The succeeding steps were the same as in the migration assay described above.

Tumorigenesis in xenografts. After $48 \mathrm{~h}$ of transfection, cells were trypsinized, stained with trypan blue, and counted. Six-week-old male nude mice were injected subcutaneously with $5 \times 10^{6}$ untreated U87 cells or HDGF-siRNA-1-treated U87 cells in $100 \mu 1$ of PBS (15 in each group). Tumor size was measured every 2 days, and 5 mice in each group were sacrificed on Days 14, 21 and 28. Tumors from sacrificed mice were weighed and then frozen in liquid nitrogen for subsequent RNA extraction. Tumor volumes were calculated as: length $x$ width $\mathrm{x}$ height.

Gene expression analysis. Total RNA was isolated from tumors using TRIzol reagent. The RNAs of siRNA-treated group and control group were pooled separately. To analyze the gene expression profile regulated by HDGF knockdown, a Whole Human Genome Oligo Microarray $(4 \times 44 \mathrm{~K}$, Agilent, CA, USA) was run by the National Engineering Center for Biochip at Shanghai (SBC; Shanghai, China). The microarray data were determined with an Agilent Microarray Scanner System and analyzed using the Agilent Feature Extraction software by SBC. Up- or downregulated genes were identified with a threshold cut-off set at 2 -fold $(\log 2$ ratio $\geq 1$, or $\leq-1)$ and a false discovery rate of $5 \%$.

Statistical analysis. Statistical analyses were performed with a two-tailed Student's t-test. All experiments reported represent at least three independent replications. Data are presented as means \pm SEM. A P-value of $<0.05$ was considered as statistically significant.

\section{Results}

Expression of HDGF in glioma cell lines and downregulation of HDGF mediated by specific siRNAs. To examine HDGF expression in the cultured human glioma cells, we performed PCR and western blotting with the U87, U373, U118, SW1783 and DBTRG lines. We found that HDGF was expressed in all five glioma cell lines. The highest expression of HDGF was observed in U87 cells, and the lowest in DBTRG cells (Fig. 1A and B). Consequently, U87 cells were chosen as the target cell line for siRNA transfection.

To specifically knock down HDGF expression in U87 cells, we synthesized and evaluated two siRNAs targeting HDGF. After $48 \mathrm{~h}$ of transfection, U87 cells treated with HDGF-siRNA-1 showed a pronounced downregulation of HDGF mRNA expression relative to untreated and to negative control siRNA-treated U87 cells (Fig. 1C). At the protein 

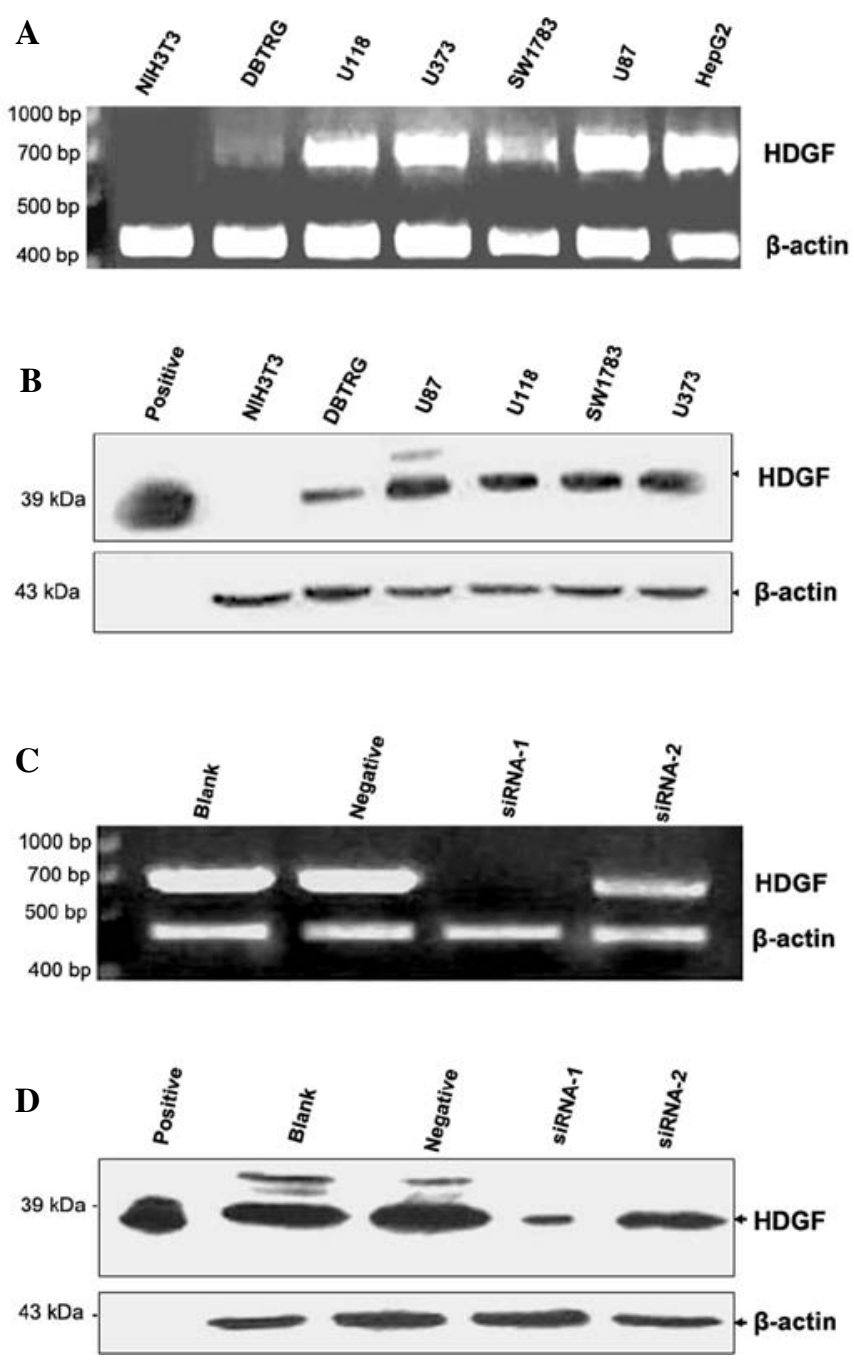

Figure 1. Expression of HDGF in glioma cell lines and suppression of HDGF mediated by HDGF-targeting siRNA. (A) PCR showing the expression of HDGF mRNA in five glioma cell lines. HepG2 and NIH 3T3 cells were used as positive and negative controls, respectively. Actin served as the internal control. (B) Western blotting of the expression of HDGF protein in glioma cell lines. Recombinant human HDGF protein was used as the positive control. (C) Downregulation of HDGF mRNA expression induced by siRNA-1. After $48 \mathrm{~h}$ of transfection, U87 cells either untreated (blank) or transfected with HDGF-siRNA-1, -siRNA-2, or scrambled RNA (negative) were harvested for PCR reaction. (D) Downregulation of HDGF protein expression mediated by HDGF siRNA-1 in U87 glioma cells, as analyzed by western blotting $72 \mathrm{~h}$ after transfection.

level, a remarkable downregulation of HDGF was confirmed in U87 cells by western blotting $72 \mathrm{~h}$ after transfection with HDGF-siRNA-1 (Fig. 1D). HDGF-siRNA-1 was selected for all in vitro and in vivo experiments.

HDGF downregulation suppressed U87 cell proliferation and colony formation. HDGF is known as a nuclear protein with growth factor activity (5). To investigate the effect of HDGF downregulation on the biological behaviors of U87 cells, we first evaluated the proliferation of U87 cells after siRNA transfection. The MTT assay results showed that HDGF knockdown reduced the growth of U87 cells compared with Lipofectamine-treated cells $(\mathrm{P}<0.05)$ (Fig. 2A). Next, we performed a soft agar colony formation assay to examine whether HDGF downregulation could inhibit anchorage-
A

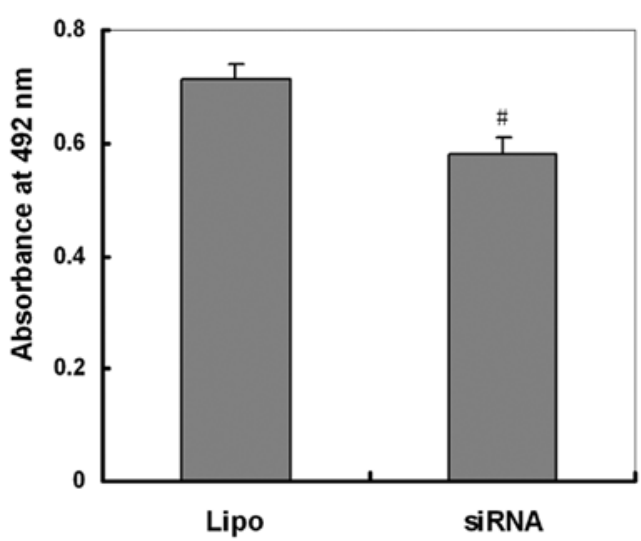

B

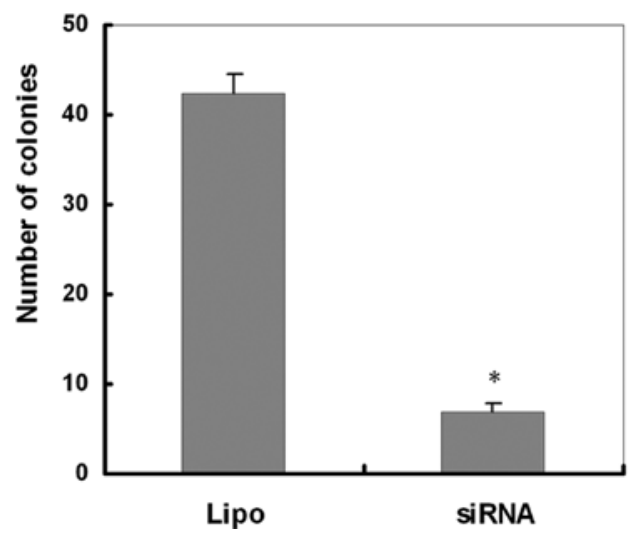

Figure 2. HDGF downregulation suppresses U87 cell proliferation and colony formation. U87 cells were transfected with Lipofectamine (Lipo) or HDGFsiRNA-1 (siRNA). (A) MTT absorbance was measured for cell proliferation. (B) Soft agar colony formation assay. Twenty-four hours after transfection, U87 cells were seeded in $0.3 \%$ agar containing DMEM and 10\% FBS. After two weeks, the number of colonies with $>50$ cells was counted using an inverted microscope. Data represent the means \pm SEM of three independent experiments. ${ }^{\text {}} \mathrm{P}<0.05$; ${ }^{\text {}} \mathrm{P}<0.01$, compared with the Lipofectamine-treated group.

independent proliferation of U87 cells. As shown in Fig. 2B, the number of colonies of U87 cells transfected with HDGFsiRNA-1 (7士0.816) were significantly fewer than the colonies of cells transfected with Lipofectamine alone (42.5 \pm 2.041 ; $\mathrm{P}<0.01)$.

Downregulation of HDGF inhibited the migration and in vitro invasion of U87 cells. To determine the effects of HDGF downregulation on U87 cell mobility, we performed a transwell migration assay. After $6 \mathrm{~h}$, seeded cells that migrated to the lower face of the membrane were stained and counted in 5 random fields. The average number of migrated cells in the HDGF-siRNA-1-treated group $(23.4 \pm 1.288)$ was significantly less than that of the Lipofectamine-treated group (59.4 \pm 3.326 ; $\mathrm{P}<0.001$ ) (Fig. 3).

We also compared the in vitro invasive ability of Lipofectamine-treated and siRNA-treated U87 cells using a Matrigel invasion assay. As shown in Fig. 3, the average number of invasive U87 cells significantly decreased after HDGF knockdown $(\mathrm{P}<0.001)$. These data demonstrated that HDGF downregulation significantly inhibited the migration and invasion of U87 cells. 
A
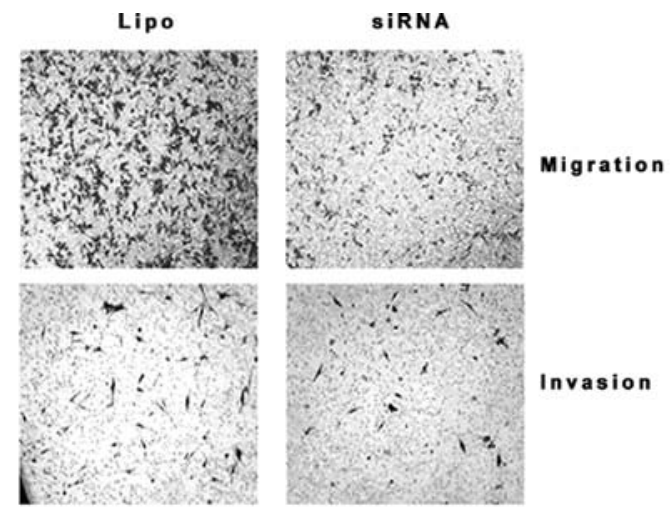

B

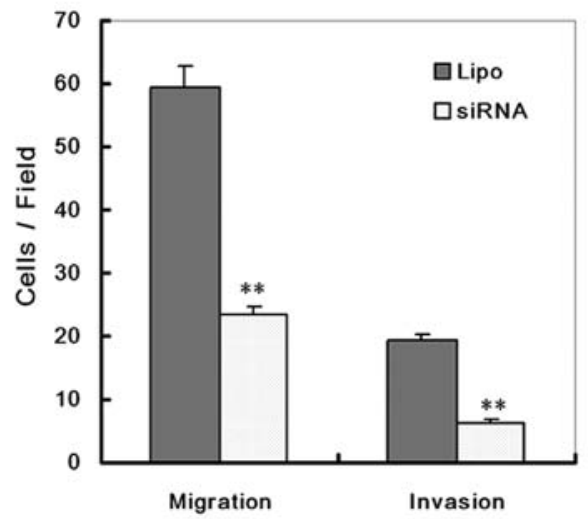

Figure 3. HDGF downregulation inhibits migration and invasion of U87 cells in vitro. U87 cells were transfected with Lipofectamine (Lipo) or with HDGF-siRNA-1 (siRNA). Cells were added into the upper chamber and then placed into wells containing $800 \mu 1$ of NIH 3T3 conditioned medium in the lower chamber. After incubation for $6 \mathrm{~h}$ (or $24 \mathrm{~h}$ for the invasion assay), the migrated cells (or invasive cells) were fixed, stained, and counted in five randomly selected fields. (A) Representative photos of migration and invasion are shown (magnification, $x 200$ ). (B) Statistical analysis of three independent experiments. Data represent the means \pm SEM. ${ }^{* *} \mathrm{P}<0.001$, compared with the Lipofectamine-treated group.

HDGF downregulation inhibited the tumorigenesis of U87 cells in vivo. To further define the role of HDGF in the tumorigenesis of U87 cells in vivo, nude mice were inoculated with either untreated or HDGF-siRNA-1-treated U87 cells. The xenografts were monitored every 2 days after inoculation, and tumor growth was quantified by tumor weight and tumor volume. As shown in Fig. 4A and B, the tumor volumes increased more slowly in the siRNA-treated group. Consistently, the tumor weight of the siRNA-treated group was lower than that of the control group: $60.89 \pm 11.422 \mathrm{mg}$ vs. $130.92 \pm 16.399 \mathrm{mg}(\mathrm{P}=0.0096)$ on Day 14 , and $113.62 \pm 10.827 \mathrm{mg}$ vs. $194.36 \pm 23.761 \mathrm{mg}(\mathrm{P}=0.011)$ on Day 21 (Fig. 4C). Given these findings, our data indicate that HDGF downregulation resulted in tumorigenesis inhibition of U87 cells in vivo.

Downregulation of HGF/SF expression induced by HDGF knockdown. To explore the possible mechanism by which HDGF affects the tumorigenesis of U87 glioma cells, we carried out oligonucleotide microarray analysis on tumor samples derived from siRNA-treated and untreated U87 cells on Day 21. When the criterion of significant mRNA level change was set at 5 -fold or above, the analysis showed that 6 genes were upregulated and 13 genes were downregulated

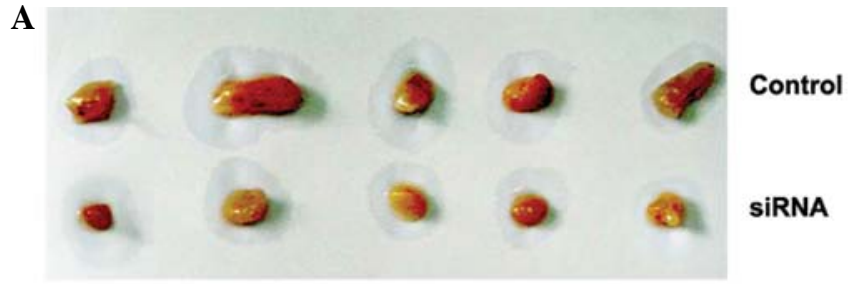

B

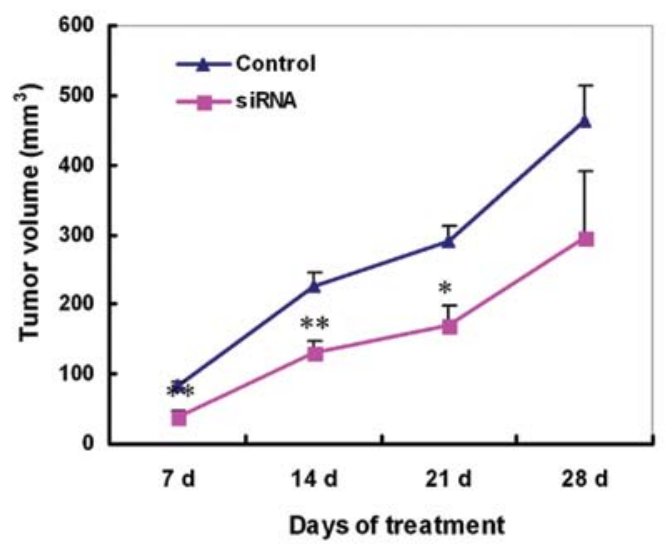

C

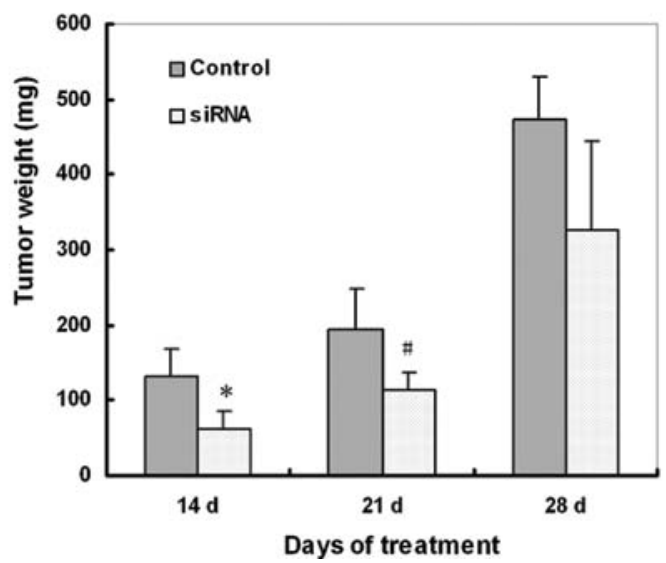

Figure 4. HDGF downregulation inhibits tumorigenesis of U87 cells in vivo Nude mice were injected with untreated (control) or HDGF-siRNA-1-treated (siRNA) U87 cells. Tumor size was measured every 2 days, and five mice in each group were sacrificed on Days 14, 21 and 28, respectively. (A) Tumors taken from each group on Day 21. (B) Tumor growth curves. Data represent the means \pm SEM ( $n=15$ on Days 7 and $14 ; n=10$ on Day $21 ; n=5$ on Day 28 in each group ). ${ }^{* *} \mathrm{P}<0.001,{ }^{*} \mathrm{P}<0.01$, compared with the control group. (C) Tumor weight on Days 14,21 and 28. Data represent the means \pm SEM (n=5). ${ }^{*} \mathrm{P}<0.01,{ }^{\#} \mathrm{P}<0.05$, compared with the control group.

(Table I). HGF/SF, which is important in glioma progression and prognosis, was found among the downregulated genes. To verify the microarray result, we examined the expression of HGF/SF in HDGF-siRNA-1-treated and untreated U87 cells. A431 cells, which rarely express HGF/SF, were used as the negative control (18). As shown in Fig. 5, both HGF/SF mRNA and protein expression were remarkably downregulated after HDGF knockdown.

\section{Discussion}

A number of clinical investigations have shown that HDGF is overexpressed in a variety of human cancers and correlates with increased malignancy and poor outcome (10-13). Some 
Table I. Gene expression profiles regulated by helatomaderived growth factor (HDGF) knockdown.

\begin{tabular}{llc}
\hline Chromosome location & Gene symbol & Log2 ratio \\
\hline $10 \mathrm{q} 22$ & CDH23 & 3.143 \\
$4 \mathrm{q} 24$ & CXXC4 & 2.664 \\
$11 \mathrm{p} 13$ & $C D 44$ & 2.57 \\
$7 \mathrm{q} 21$ & ADAM22 & 2.501 \\
$21 \mathrm{q} 22$ & UBB & 2.341 \\
$17 \mathrm{p} 11$ & COL6A1 & 2.331 \\
$4 \mathrm{q} 13$ & CXCL2 & -3.248 \\
$19 \mathrm{p} 13$ & SMARCA4 & -3.239 \\
$17 \mathrm{q} 21$ & RAB5C & -3.048 \\
$10 \mathrm{q} 24$ & NFKB2 & -2.947 \\
$1 \mathrm{p} 32$ & JUN & -2.942 \\
$4 \mathrm{p} 15$ & MED28 & -2.8 \\
$20 \mathrm{q} 13$ & LAMA5 & -2.758 \\
$14 \mathrm{q} 24$ & ADAM21 & -2.745 \\
$10 \mathrm{q} 24$ & LCOR & -2.707 \\
$20 \mathrm{q} 13$ & EDN3 & -2.562 \\
$8 \mathrm{q} 24$ & TNFRSF11B & -2.524 \\
$7 \mathrm{q} 21$ & HGF & -2.413 \\
9p21 & CDKN2A & -2.362 \\
\hline
\end{tabular}

A

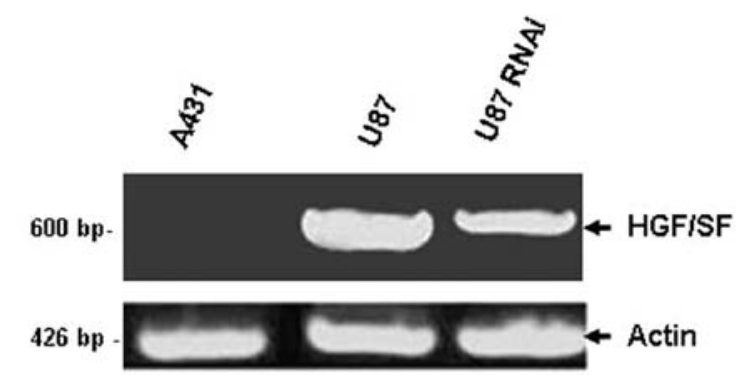

B

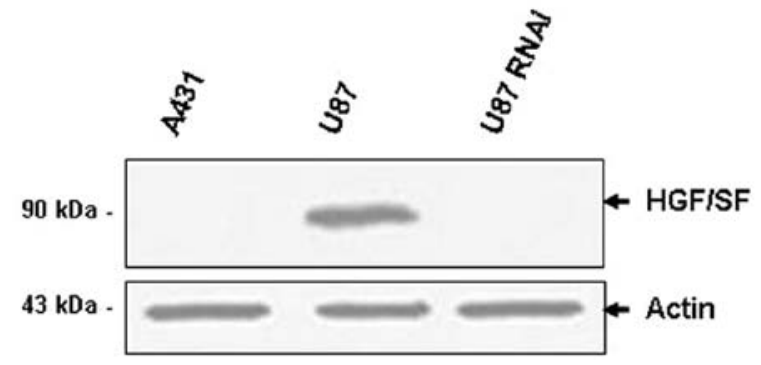

Figure 5. Downregulation of HGF/SF expression induced by HDGF knockdown. The expression of HGF/SF was analyzed at the mRNA (A) and protein level (B) in both untreated (U87) and HDGF-siRNA-1-treated (U87 RNAi) U87 cells. A431 cells (A431) were used as the negative control, and actin served as the internal control.

research suggests that HDGF is involved in aggressive tumor behaviors such as progression, angiogenesis, metastasis and recurrence $(11,19-21)$. We previously found that HDGF was overexpressed in glioma tissues and that the more advanced the stage, the higher the expression level of HDGF (14). However, it remains unclear how HDGF affects the biological behavior of glioma cells.

As a mitogenic factor, the proliferative activity of HDGF has been extensively investigated $(22,23)$. It has been reported that HDGF knockdown by antisense oligomers repressed the proliferation of HepG2 cells in either an anchorage-dependent or -independent manner (24). We found that downregulation of HDGF expression significantly inhibited the proliferation of both DU145 prostate cancer cells and MCF7 breast cancer cells (25). In non-small cell lung cancer (NSCLC) cells, downregulation of HDGF had minimal effect on anchoragedependent cell proliferation, but it significantly reduced anchorage-independent cell growth (16).

To test the effects of HDGF knockdown on the growth of U87 cells, we compared the proliferation of siRNA-treatedand Lipofectamine-treated U87 cells using an MTT assay and a soft agar colony formation assay. We found that although the proliferation of U87 cells after HDGF downregulation was suppressed and a statistically significant difference was obtained $(\mathrm{P}=0.032)$, HDGF seemed not to be a potent factor for anchorage-dependent cell growth in these cells (Fig. 2A). The colony formation results showed that U87 cells transfected with HDGF-siRNA-1 produced significantly fewer colonies $(7 \pm 1.4)$, only $16.5 \%$ of that from cells transfected with Lipofectamine alone (42.5 \pm 2.5$)$ (Fig. 2B). This result suggested that downregulation of HDGF profoundly inhibited the anchorage-independent growth of U87 glioma cells, with a minor effect on anchorage-dependent growth. The published results and our present data indicate that the role of endogenous HDGF in cell growth is complex and may depend on tissue type, cell type, and other conditions.

The ability to form colonies in soft agar indicates the potential for cancer cell invasion and in vivo tumorigenesis (26). In gastric cancer and lung cancer, it has been shown that HDGF expression is closely related to the depth of invasion and to the status of lymphoid metastases and distant metastases $(10,11)$. We examined whether HDGF was involved in the migration and invasion of U87 glioma cells. In our results, as expected, the average number of migrating cells decreased in the siRNA-treated group, and the number of cells penetrating through the artificial basement membrane in a Matrigel assay decreased significantly, showing that HDGF downregulation markedly inhibited the migration and invasion of U87 cells in vitro.

It has been reported that HDGF participates in the development of liver cancer and gastric cancer $(27,28)$. Overexpression of HDGF results in tumorigenesis by NIH 3T3 cells, and dowregulation of HDGF inhibits the tumorigenicity of NSCLC cells in vivo $(10,20)$. Therefore, we investigated the effect of HDGF downregulation on the tumorigenesis of U87 cells in a subcutaneous model. siRNA-treated xenografts grew more slowly than untreated U87 xenografts, especially in the early stages (Fig. 4B and C), which suggested that HDGF downregulation impaired the tumorigenesis of U87 cells in vivo. Although both the tumor volume and the tumor weight of the siRNA-treated group were still lower than those of the control group on Day 28, there was no significant difference between the two groups at that time point, which may be due to the time-limited effect of a transient transfection of siRNA (29). 
We also compared the gene expression profiles of the xenografts from HDGF-siRNA-1 treated and untreated cells. Microarray data showed that the genes regulated by HDGF in glioma are different from those in prostate cancer (25) or NSCLC cells (16) (Table I). We found that the gene for $H G F /$ $S F$ was downregulated (Table I), which was further confirmed by PCR and western blotting (Fig. 5). To our knowledge, this is the first report to show a relationship between HDGF and $\mathrm{HGF} / \mathrm{SF}$ in glioma.

$\mathrm{HGF} / \mathrm{SF}$ is a unique multifunctional factor because it can stimulate invasive cell growth. It affects most of the functions of tumor progression such as cell dissociation, motility and migration, proliferation and angiogenesis (30). HGF/ $\mathrm{SF}$ is strongly implicated as a regulator of tumor progression and metastasis in a wide variety of human cancers, including gliomas $(31,32)$, and is the most potent stimulator of glioma cell migration (33). Earlier reports and our current data revealed the similarity of biological functions between HDGF and HGF/SF in glioma. In addition, HDGF knockdown significantly downregulated HGF/SF expression. We hypothesize that the inhibitory effects of knocking down HDGF in glioma cells may be due to the reduction of HGF/SF or, from a different perspective, HDGF can be considered as an upstream molecule in a signaling cascade in which that has HGF/SF is one of the downstream molecules. This topic needs to be further investigated.

In summary, our present study shows that the downregulation of HDGF significantly inhibits the malignant phenotypes of U87 cells in terms of colony formation, migration, and invasion in vitro, as well as tumorigenesis in vivo. The data strongly suggest that selective targeting of HDGF may offer a new option for glioma molecular therapy.

\section{Acknowledgements}

We thank Mr. David Nadziejka for review and editing of the manuscript. This study was supported by the Nature Science Foundation of Jiangsu (Project No. BK2011767), and was partially supported by the Jay and Betty Van Andel Foundation.

\section{References}

1. Wen PY and Kesari S: Malignant gliomas in adults. N Engl J Med 359: 492-507, 2008.

2. Van Meir EG, Hadjipanayis CG, Norden AD, Shu HK, Wen PY and Olson JJ: Exciting new advances in neuro-oncology: the avenue to a cure for malignant glioma. CA Cancer J Clin 60: 166-193, 2010.

3. Huang TT, Sarkaria SM, Cloughesy TF and Mischel PS: Targeted therapy for malignant glioma patients: lessons learned and the road ahead. Neurotherapeutics 6: 500-512, 2009.

4. Nakamura H, Kambe H, Egawa T, et al: Partial purification and characterization of human hepatoma-derived growth factor. Clin Chim Acta 183: 273-284, 1989.

5. Everett AD and Bushweller J: Hepatoma derived growth factor is a nuclear targeted mitogen. Curr Drug Targets 4: 367-371, 2003.

6. Abouzied MM, Baader SL, Dietz F, Kappler J, Gieselmann V and Franken S: Expression patterns and different subcellular localization of the growth factors HDGF (hepatoma-derived growth factor) and HRP-3 (HDGF-related protein-3) suggest functions in addition to their mitogenic activity. Biochem J 378: 169-176, 2004.
7. Oliver JA and Al-Awqati Q: An endothelial growth factor involved in rat renal development. J Clin Invest 102: 1208-1219, 1998.

8. Everett AD: Identification, cloning, and developmental expression of hepatoma-derived growth factor in the developing rat heart. Dev Dyn 222: 450-458, 2001.

9. Enomoto H, Yoshida K and Kishima Y, et al: Hepatomaderived growth factor is highly expressed in developing liver and promotes fetal hepatocyte proliferation. Hepatology 36: 1519-1527, 2002.

10. Ren H, Tang X, Lee J, et al: Expression of hepatoma-derived growth factor is a strong prognostic predictor for patients with early-stage non-small-cell lung cancer. J Clin Oncol 22: 3230-3237, 2004

11. Yamamoto S, Tomita Y, Hoshida Y, et al: Expression of hepatoma-derived growth factor is correlated with lymph node metastasis and prognosis of gastric carcinoma. Clin Cancer Res 12: 117-122, 2006.

12. Yoshida K, Tomita Y, Okuda Y, et al: Hepatoma-derived growth factor is a novel prognostic factor for hepatocellular carcinoma. Ann Surg Oncol 13: 159-167, 2006.

13. Uyama H, Tomita Y, Nakamura $\mathrm{H}$, et al: Hepatoma-derived growth factor is a novel prognostic factor for patients with pancreatic cancer. Clin Cancer Res 12: 6043-6048, 2006.

14. Zhang A, Long W, Guo Z, et al: Development and clinical evaluation of a multi-purpose $\mathrm{mAb}$ and a sandwich ELISA test for hepatoma-derived growth factor in lung cancer patients. J Immunol Methods 355: 61-67, 2010.

15. Cao B, Su Y, Oskarsson M, Zhao P, Kort EJ, Fisher RJ, Wang LM and Vande Woude GF: Neutralizing monoclonal antibodies to hepatocyte growth factor/scatter factor $(\mathrm{HGF} / \mathrm{SF})$ display antitumor activity in animal models. Proc Natl Acad Sci USA 98: 7443-7448, 2001.

16. Zhang J, Ren H, Yuan P, Lang W, Zhang L and Mao L: Down-regulation of hepatoma-derived growth factor inhibits anchorage-independent growth and invasion of non-small cell lung cancer cells. Cancer Res 66: 18-23, 2006.

17. Shi H, Wang J, Dong F, Wang X, Li H and Hou Y: The effect of proteoglycans inhibited by RNA interference on metastatic characters of human salivary adenoid cystic carcinoma. BMC Cancer 9: 456-470, 2009

18. Matsuki A, Yamamoto S and Nakagami H: No influence of tumor growth by intramuscular injection of hepatocyte growth factor plasmid DNA: safety evaluation of therapeutic angiogenesis gene therapy in mice. Biochem Biophys Res Commun 315: 59-65, 2004.

19. Bernard K, Litman E, Fitzpatrick JL, et al: Functional proteomic analysis of melanoma progression. Cancer Res 63: 6716-6725, 2003.

20. Okuda Y, Nakamura H, Yoshida K, et al: Hepatoma-derived growth factor induces tumorigenesis in vivo through both direct angiogenic activity and induction of vascular endothelial growth factor. Cancer Sci 94: 1034-1041, 2003.

21. Yamamoto S, Tomita Y, Hoshida Y, et al: Expression level of hepatoma-derived growth factor correlates with tumor recurrence of esophageal carcinoma. Ann Surg Oncol 14: 2141-2149, 2007.

22. Everett AD, Stoops T and McNamara CA: Nuclear targeting is required for hepatoma-derived growth factor-stimulated mitogenesis in vascular smooth muscle cells. J Biol Chem 276: 37564-37568, 2001.

23. Kishima Y, Yamamoto H, Izumoto Y, et al: Hepatoma-derived growth factor stimulates cell growth after translocation to the nucleus by nuclear localization signals. J Biol Chem 277: 10315-10322, 2002.

24. Kishima Y, Yoshida K, Enomoto H, et al: Antisense oligonucleotides of hepatoma-derived growth factor (HDGF) suppress the proliferation of hepatoma cells. Hepatogastroenterology 49: 1639-1644, 2002.

25. Guo Z, He Y, Wang S, Zhang A, Zhao P, Gao C and Cao B: Various effects of hepatoma-derived growth factor on cell growth, migration and invasion of breast cancer and prostate cancer cells. Oncol Rep 26: 511-517, 2011.

26. Masri J, Bernath A, Martin J, Jo OD, Vartanian R, Funk A and Gera J: mTORC2 activity is elevated in gliomas and promotes growth and cell motility via overexpression of rictor. Cancer Res 67: 11712-11720, 2007.

27. Yoshida K, Nakamura H, Okuda Y, et al: Expression of hepatomaderived growth factor in hepatocarcinogenesis. J Gastroenterol Hepatol 18: 1293-1301, 2003. 
28. Mao J, Xu Z, Fang Y, et al: Hepatoma-derived growth factor involved in the carcinogenesis of gastric epithelial cells through promotion of cell proliferation by Erk1/2 activation. Cancer Sci 99: 2120-2127, 2008.

29. Malecová $\mathrm{B}$ and Morris KV: Transcriptional gene silencing through epigenetic changes mediated by non-coding RNAs. Curr Opin Mol Ther 12: 214-222, 2010.

30. Desiderio MA: Hepatocyte growth factor in invasive growth of carcinomas. Cell Mol Life Sci 64: 1341-1354, 2007.
31. Gao C and Vande Woude GF: HGF/SF-Met signaling in tumor progression. Cell Res 15: 49-51, 2005.

32. Abounader R and Laterra J: Scatter factor/hepatocyte growth factor in brain tumor growth and angiogenesis. Neuro Oncol 7: 436-451, 2005.

33. Brockmann MA, Ulbricht U, Gruner K, Fillbrandt R, Westphal M and Lamszus K: Glioblastoma and cerebral microvascular endothelial cell migration in response to tumor-associated growth factors. Neurosurgery 52: 1391-1399, 2003. 